\title{
Process and Product Innovation in a Vertically Differentiated Monopoly
}

\author{
Luca Lambertini and Raimondello Orsini \\ Department of Economics \\ University of Bologna \\ Strada Maggiore 45 \\ 40125 Bologna, Italy \\ fax: +39-051-2092664 \\ e-mail: lamberti@spbo.unibo.it \\ e-mail: orsini@spbo.unibo.it
}

January 27, 2000

\begin{abstract}
We analyse product and process innovations in a monopoly model with vertical differentiation. The incentive towards both kinds of innovation is always larger under social planning than under monopoly. Therefore, the comparison between the two regimes' welfare performance should account for the possibility that the planner and the monopolist adopt different technologies and supply different product ranges.
\end{abstract}

JEL Classification: L12, O31

Keywords: product innovation, process innovation, vertical differentiation 


\section{Introduction}

It is commonly observed that firms activate R\&D portfolios including several innovation projects (Bhattacharya and Mookherjee, 1986; Dasgupta and Maskin, 1987). R\&D can be conducted along two different directions, namely, process and product innovation. The literature usually treats the two kinds of innovation separately, a relevant exception being Rosenkranz (1996).

Our aim is to show that jointly investigating product and process innovations in a monopoly model with vertical differentiation can reshape some of the established wisdom in this field, dating back to Mussa and Rosen (1978). In particular, we show that the social incentive towards both kinds of innovation is always larger than the private incentive characterising the profit-seeking monopolist. In turn, this implies that the efficiency comparison between the two regimes should take into account that they may differ with respect to both product range and technology.

The remainder of the note is organised as follows. The monopolist's optimal behaviour is laid out in section 2 . Section 3 investigates the inefficiency of monopoly as against the social optimum.

\section{Monopoly optimum}

Consider a simplified version of the vertical differentiation setting by Mussa and Rosen (1978). At the outset, a monopolist sells a single good of quality $q$ at price $p$. Production involves variable costs only:

$$
C=q^{2} x
$$

where $x$ is the output level. Consumers are uniformly distributed with density 1 over the interval $[0, \bar{\theta}]$. Hence, the total size of the market is $\bar{\theta}$. Parameter $\theta$ identifies each consumer's marginal willingness to pay. Each individual maximizes his net surplus, which is $U=\theta q-p \geq 0$ if he buys, otherwise it is nil. Accordingly, the demand function is $x=\bar{\theta}-p / q$. Since $p / q>0$, the market is only partially served. With a single product and the technology initially available, the monopolist's objective function is $\Pi_{s q}=\left(p-q^{2}\right) x$, where subscript $s q$ stands for status quo and refers to the initial situation where the monopolist is a single-product firm with production costs as in (1). Equilibrium profits are $\Pi_{s q}^{*}=\bar{\theta}^{3} / 27$. Optimal quality is $q^{*}=\bar{\theta} / 3$ (for computational details, see Lambertini, 1997). 
In modelling innovation, we assume uncertainty away. R\&D activity can be carried out along two directions, namely, product and process innovation. Product innovation can be illustrated as follows. When a second variety is introduced, the monopolist finds it optimal to modify the existing quality, supplying qualities $q_{H}$ and $q_{L}$, with $q_{H}>q_{L}$. Restructuring production entails a cost $\mu>0$. Process innovation consists in changing the cost function from $q^{2} x$ to $\tau q^{2} x$, with $\tau \in(0,1)$. This cost abatement can be achieved by investing $k$, regardless of the number of varieties being supplied. In general, the monopolist may pursue both innovations. With two products, market demands are $x_{H}=\bar{\theta}-\left(p_{H}-p_{L}\right) /\left(q_{H}-q_{L}\right)$ and $x_{L}=\left(p_{H}-p_{L}\right) /\left(q_{H}-q_{L}\right)-$ $p_{L} / q_{L}$. The objective function is defined as follows:

$$
\Pi_{2 \tau}=\left[p_{H}-\tau q_{H}^{2}\right] x_{H}+\left[p_{L}-\tau q_{L}^{2}\right] x_{L}-k-\mu
$$

Subscript $2 \tau$ indicates that the monopolist has introduced a second variety and lowered unit cost to $\tau q^{2}$ for both varieties. Maximising (2) w.r.t. prices and qualities yields $\left\{p_{H}^{*}=7 \bar{\theta}^{2} /(25 \tau) ; p_{L}^{*}=3 \bar{\theta}^{2} /(25 \tau) ; q_{H}^{*}=2 \bar{\theta} /(5 \tau) ; q_{L}^{*}=\right.$ $\bar{\theta} /(5 \tau)\}$ (see Lambertini, 1997). Profits (2) simplify to $\Pi_{2 \tau}^{*}=\bar{\theta}^{3} /(25 \tau)-k-\mu$. Alternatively, the monopolist may choose to pursue either product or process innovation only. In the case of product innovation, equilibrium profits are $\Pi_{21}^{*}=\bar{\theta}^{3} / 25-\mu$. When process innovation is activated, equilibrium profits are $\Pi_{1 \tau}^{*}=\bar{\theta}^{3} /(27 \tau)-k$.

The optimal portfolio of innovations for the monopolist is summarised in the following:

Theorem 1 The necessary condition for both process and product innovations to be carried out in equilibrium is $k+\mu<\bar{\theta}^{3}(27-25 \tau) /(675 \tau)$.

Sufficient conditions are:

I. $k<\bar{\theta}^{3}(1-\tau) /(27 \tau)$ and $\mu<2 \bar{\theta}^{3} /(675 \tau)$, or

II. $k<\bar{\theta}^{3}(1-\tau) /(25 \tau)$ and $\mu<2 \bar{\theta}^{3} / 675$.

If $k<\bar{\theta}^{3}(1-\tau) /(27 \tau)$ and $\mu>2 \bar{\theta}^{3} /(675 \tau)$, the monopolist remains a single-product firm and activates cost-reducing RED for the existing variety.

If $\mu<2 \bar{\theta}^{3} / 675$ and $k>\bar{\theta}^{3}(1-\tau) /(25 \tau)$, the monopolist introduces the second variety, keeping the existing technology unmodified. 
Proof. To prove the theorem, we proceed in steps. Consider first the incentive to conduct cost-reducing $\mathrm{R} \& \mathrm{D}$ with a single variety. We have that

$$
\Pi_{1 \tau}^{*}>\Pi_{s q}^{*} \text { if } k<\frac{\bar{\theta}^{3}(1-\tau)}{27 \tau}
$$

The incentive to carry out product innovation with the existing technology is summarised by the following:

$$
\Pi_{21}^{*}>\Pi_{s q}^{*} \text { if } \mu<\frac{2 \bar{\theta}^{3}}{675}
$$

Then, examine the incentive to reduce production costs, provided the monopolist is willing to introduce a second variety:

$$
\Pi_{2 \tau}^{*}>\Pi_{21}^{*} \text { if } k<\frac{\bar{\theta}^{3}(1-\tau)}{25 \tau}
$$

The incentive to expand product variety, provided the monopolist finds it profitable to reduce unit costs, is summarised by:

$$
\Pi_{2 \tau}^{*}>\Pi_{1 \tau}^{*} \text { if } \mu<\frac{2 \bar{\theta}^{3}}{675 \tau}
$$

Since $\tau \in(0,1)$, condition (6) is less demanding than condition (4). Therefore, in evaluating sufficiency, we take into account (6). Finally, observe that

$$
\Pi_{2 \tau}^{*}>\Pi_{s q}^{*} \text { if } k+\mu<\frac{\bar{\theta}^{3}(27-25 \tau)}{675 \tau}
$$

This is a necessary but not sufficient condition for the firm to undertake both innovations.

\section{Welfare appraisal}

We now proceed to evaluate the social damage associated with the monopoly equilibrium. To this aim, consumer surplus, with one or two varieties, can be computed as follows:

$$
C S_{1}=\int_{p / q}^{\bar{\theta}}(\theta q-p) d \theta
$$




$$
C S_{2}=\int_{a}^{\bar{\theta}}\left(\theta q_{H}-p_{H}\right) d \theta+\int_{b}^{a}\left(\theta q_{L}-p_{L}\right) d \theta
$$

where $a=\left(p_{H}-p_{L}\right) /\left(q_{H}-q_{L}\right)$ and $b=p_{L} / q_{L}$. Social welfare is the sum of profits and consumer surplus. Now define equilibrium social welfare and profits, gross of innovation costs, as $\widehat{S W}^{*}$ and $\widehat{\Pi}^{*}$, respectively. Straightforward calculations show that, in general, $\widehat{S W}^{*}=3 \widehat{\Pi}^{*} / 2$, that is, gross social welfare in the monopoly optimum is $50 \%$ larger than gross monopoly profits. This suffices to prove that the social incentive to innovate is $50 \%$ larger than the private incentive.

Now, turn to the situation where the planner controls prices and qualities and looks for the first best. To evaluate his incentives towards innovation, we calculate social welfare levels associated with one and two varieties, respectively, under marginal cost pricing. Moreover, on the basis of Spence (1975), we know that the planner supplies the same qualities as the monopolist. As a result, we have:

$$
S W_{1 \tau}^{S P}=\frac{2 \bar{\theta}^{3}}{27 \tau}-k ; S W_{2 \tau}^{S P}=\frac{2 \bar{\theta}^{3}}{25 \tau}-k-\mu
$$

where superscript $S P$ stands for social planning. The relevant magnitudes when process innovation is not undertaken, obtain from (10) by setting $k=0$ and $\tau=1$. In any of the four possible cases, $\widehat{S W}^{S P}=2 \widehat{\Pi}^{*}$, i.e., gross social welfare in the social optimum is twice as large as gross producer surplus in the monopoly optimum. This leads to the following:

Proposition 1 Evaluate social planning against the monopoly optimum. The social incentive to innovate in the former is twice as large as the private incentive in the latter.

As the proof entails simple algebra, we just illustrate the case of process innovation with one variety. The social incentive is measured by $S W_{1 \tau}^{S P}-$ $S W_{s q}^{S P}$, which is positive if $k<2 \bar{\theta}^{3}(1-\tau) /(27 \tau)$. The r.h.s. of this inequality is twice as large as the r.h.s. in condition (3). The same holds in all other cases as well.

The above proposition has some relevant bearings on the assessment of the social inefficiency of monopoly. In the existing literature (Mussa and Rosen, 1978; Maskin and Riley, 1984; Champsaur and Rochet, 1989, inter alia), this evaluation is performed for a given technology, under the additional 
assumption that expanding the product range is costless, i.e., $\mu=0$. On these bases, one reaches the conclusion that the monopolist's quality range is wider than the socially optimal one, in that the monopolist aims at inducing: self-discrimination among customers. Our approach allows us to raise two objections to the acquired wisdom. The first is that, for a given number of varieties, there are parameter ranges where the planner operates with a more efficient technology than the profit-seeking monopolist. As an example, this happens when $k \in\left(\bar{\theta}^{3}(1-\tau) /(27 \tau), 2 \bar{\theta}^{3}(1-\tau) /(27 \tau)\right)$. In this case, the monopolist does not carry out a welfare-improving process innovation. The second objection relies on the following argument. Suppose:

$$
k+\mu \in\left(\frac{\bar{\theta}^{3}(27-25 \tau)}{675 \tau}, 2 \frac{\bar{\theta}^{3}(27-25 \tau)}{675 \tau}\right)
$$

and, e.g., $S W_{1 \tau}^{S P} \in\left(S W_{s q}^{S P}, S W_{2 \tau}^{S P}\right) ; \Pi_{s q}^{*}>\max \left\{\Pi_{1 \tau}^{*}, \Pi_{21}^{*}\right\}$. If so, the planner undertakes both innovations while the monopolist remains in the status quo, since the cost of product proliferation outweighs the private benefits associated with discrimination among consumers. Therefore, the different incentives towards innovation characterising monopoly and social planning may entail that one should compare the two regimes taking into account that both product range and technology can differ in ways that the literature has overlooked so far. 


\section{References}

[1] Bhattacharya, S. and D. Mookherjee (1986), Portfolio Choice in Research and Development, RAND Journal of Economics, 17, 594-605.

[2] Champsaur, P. and J.-C. Rochet (1989), Multiproduct Duopolists, Econometrica, 57, 533-57.

[3] Dasgupta, P. and E. Maskin (1987), The Simple Economics of Research Portfolios, Economic Journal, 97, 581-95.

[4] Lambertini, L. (1997), The Multiproduct Monopolist under Vertical Differentiation: An Inductive Approach, Recherches Economiques de Louvain, 63, 109-22.

[5] Maskin, E. and J. Riley (1984), Monopoly with Incomplete Information, RAND Journal of Economics, 15, 171-196.

[6] Mussa, M. and S. Rosen (1978), Monopoly and Product Quality, Journal of Economic Theory, 18, 301-17.

[7] Rosenkranz, S. (1996), Simultaneous Choice of Process and Product Innovation, CEPR Discussion Paper n. 1321.

[8] Spence, A.M. (1975), Monopoly, Quality and Regulation, Bell Journal of Economics, 6, 417-29. 